\title{
MIGRANTS ADMITTED TO AN INPATIENT SERVICE IN PORTUGAL A SOCIODEMOGRAPHIC AND CLINICAL CHARACTERIZATION
}

B. Moura ${ }^{1,2,3}$, T. Rodrigues ${ }^{1}$, A. Miguel ${ }^{1}$, S. Ramos $^{3}$, R. Ribeiro Silva 1.

${ }^{1}$ Centro Hospitalar Vila Nova de Gaia/Espinho (CHVNG/E), Department of Psychiatry and Mental Health, Vila Nova de Gaia, Portugal. 2 EFPT Research Working Group. ${ }^{3}$ Unidade Local de Saúde da Guarda, Department of Psychiatry and Mental Health. ${ }^{4}$ Corresponding Author: barbararmoura@gmail..com

Background. It has been established that the process of migration and subsequent cultural adjustment play a key role in an individual's mental health. Migration can contribute to the development of mental health disorders, with an increased risk for psychosis found across several migrant populations. Portugal is a country with a long history of emigration, but recently there has been a surge in immigration as well. Few studies on the mental health of immigrants in Portugal are available.

Aims. We aimed to characterize the sample of first generation migrants admitted to our inpatient service, from 2004-2018, from a socio-demographic and clinical standpoint.

Methods. Data was collected through a review of clinical records. A descriptive analysis was conducted.
Table 1. Vulnerability factors for mental health disorders at each stage of migration (adapted from Bhugra \& Jones, 2001)

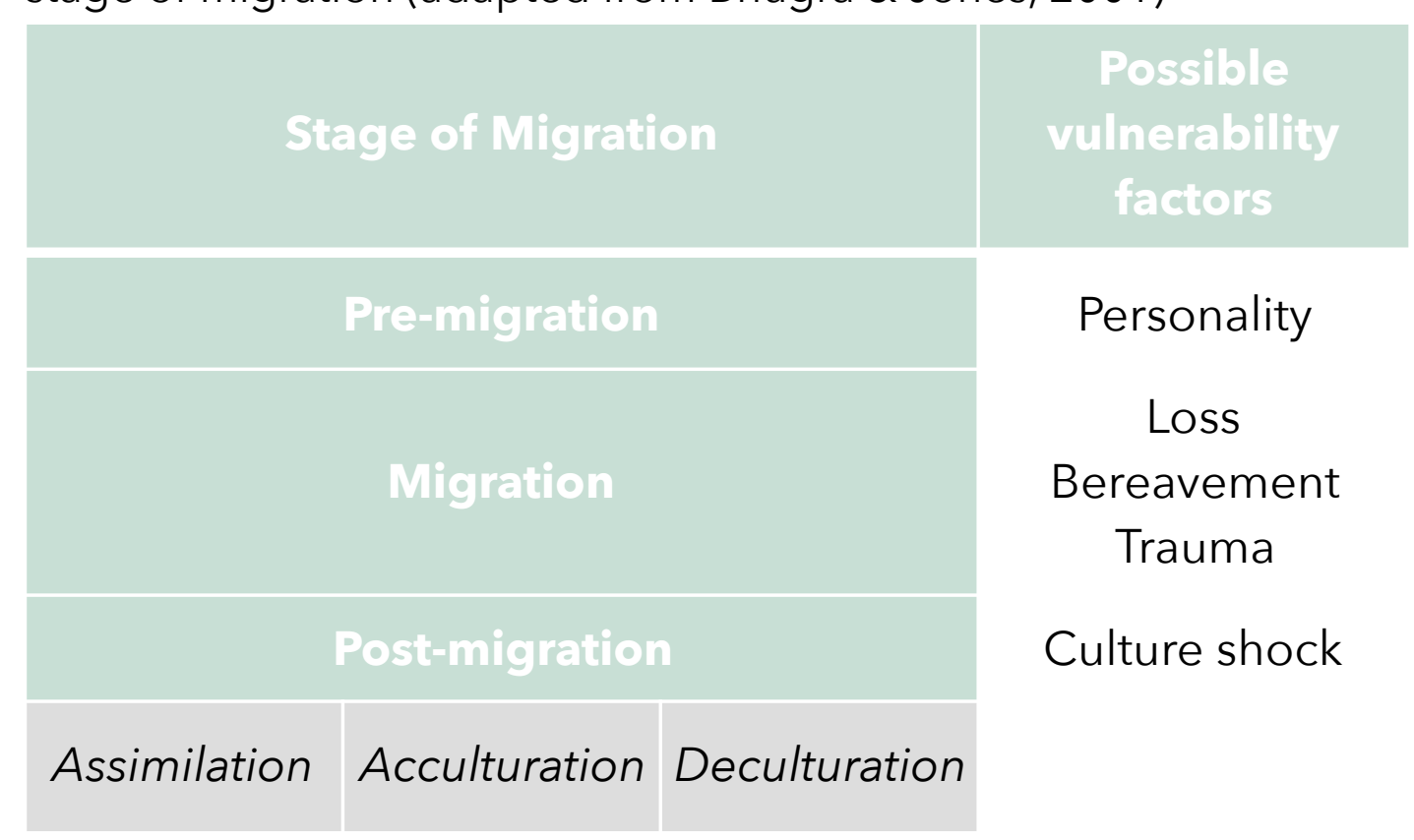

Results. The graphs below illustrate the characteristics of migrants admitted to the psychiatric inpatient service of CHVNG/E.

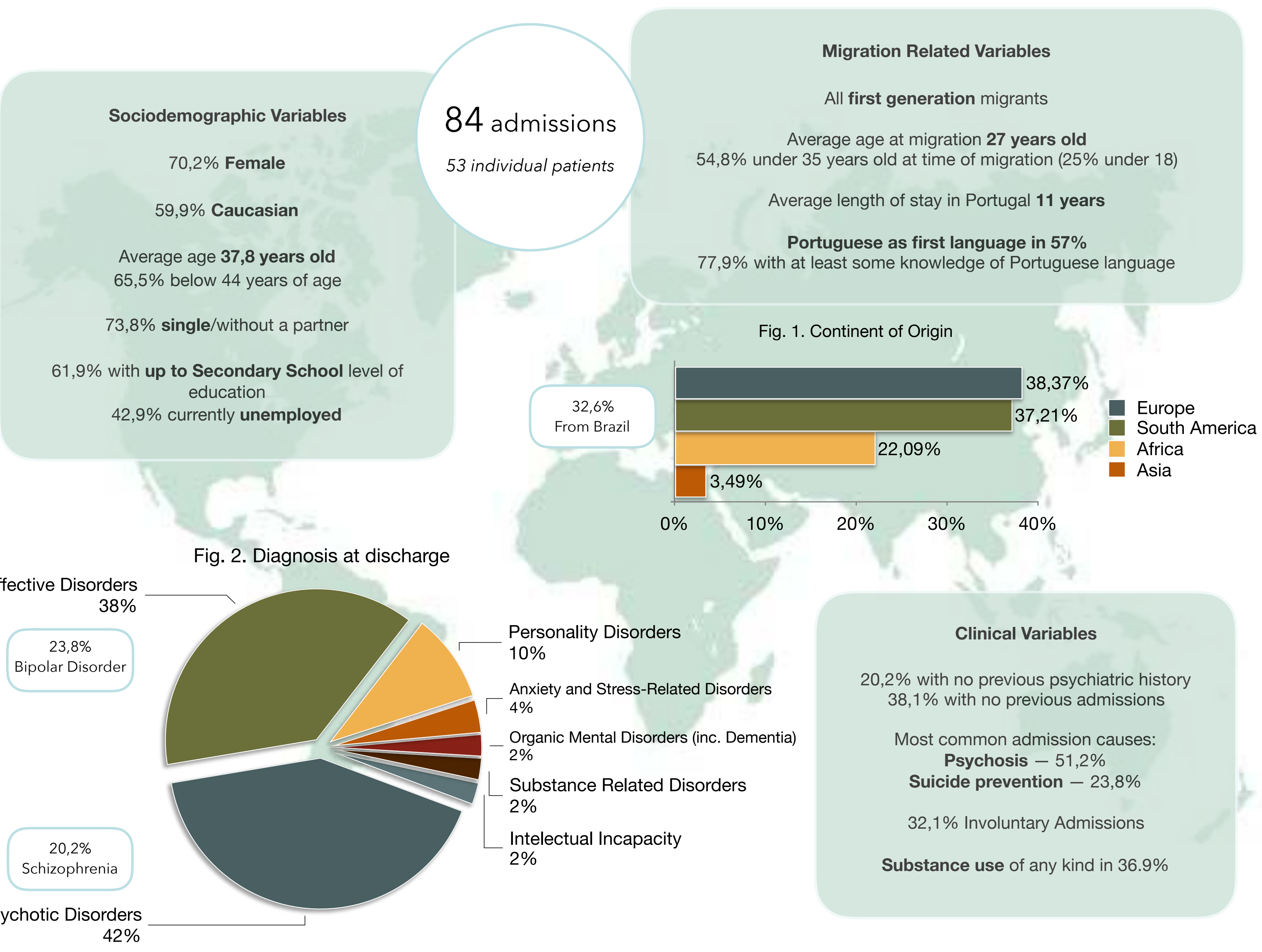

Conclusion. This study allows us to outline the profile of the migrants who are admitted to our inpatient service; they are mostly young females, of caucasian ethnicity, with a low educational background, and unemployed, which points to unfavorable socioeconomic circumstances. Many have positive factors regarding adaptation, such as a dominion of the Portuguese language. Clinically, findings such as the high rate of Psychotic Disorders and substance use are in agreement with previous studies. Further studies will help determine the care pathways of migrants after discharge from our inpatient unit, as well as their specific needs in terms of mental health care and social support structures. 